Instituto Internacional de Investigación y Desarrollo Tecnológico Educativo INDTEC, C.A.

DOI: https://doi.org/10.29394/scientific.issn.2542-2987.2017.2.4.20.351-361

OAI-PMH: http://www.indteca.com/ojs/index.php/Revista Scientific/oai

\title{
Economía Verde, Una Propuesta para Salvaguardar la Vida en el Planeta
}

\author{
Autor: Oscar Eduardo Cabrera Venot \\ Instituto Universitario de Tecnología Puerto Cabello, IUTPC \\ oscarcvenot@gmail.com \\ Puerto Cabello, Venezuela
}

\section{Resumen}

Muchas organizaciones en el mundo dedican sus esfuerzos a la presentación de planes y propuestas que permitan la conservación del planeta y sus habitantes basadas en la preservación del ambiente, y atacando específicamente la causa del calentamiento global, producto del efecto invernadero. Todos estos esfuerzos deben ser bienvenidos, las propuestas convertirse en hechos tangibles y la población mundial en pleno debe estar sensibilizada sobre su papel como individuo en este escenario. Sin embargo, detrás de todo esto existen intereses de orden económico que de manera importante afectarían las economías de naciones enteras, y es allí donde se presentan las principales trabas para la aplicación de programas conservacionistas. Este ensayo, basado en una revisión documental y en análisis de fuentes bibliográficas, se propone mostrar los principales aspectos que a criterio del autor, deben ser considerados en una eco-economía, término introducido por Brown en el año 2006, o en una economía verde, para ello se presentan algunas reflexiones sobre la economía mundial en la actualidad, para luego presentar algunas ponderaciones sobre las variables que están estresando al planeta en cuanto a cambio climático y las bases biológicas. Se consideran los aspectos relacionados con un nuevo orden económico mundial en cuanto a combustibles, nuevos materiales, alimentación de la población, protección de los bosques y rediseño de las ciudades. Finalmente, se reflexionó sobre la estabilización de la población mundial, la reglas para reestructurar la economía y aceleración del proceso de transición.

Palabras clave: economía; ecología; preservación de vida. 


\title{
Green Economy, A Proposal to Safeguard the Life on the Planet
}

\begin{abstract}
Many organizations around the world dedicate their efforts to the presentation of plans and proposals for the conservation of the planet and its inhabitants based on the preservation of the environment, and specifically attacking the causes of global warming, inflicted by greenhouse effect. All these efforts are to be welcomed, the proposals become tangible facts and in the world population must be sensitized on their role as an individual in this scenario. But behind all this there are interests of economic order that significantly affect the economies of entire nations, and that is where the main obstacles to the implementation of conservation programs are presented. This essay, based on a literature review and analysis of literature sources, aims to show the main aspects to the author, should be considered in an eco-economy, a term introduced by Brown in 2006, or a green economy, for that some reflections on the present world economy are presented, then present some considerations on the variables that are stressing the planet on climate change and biological bases. Then some aspects of a new world economic order as to fuels, new materials, feeding the population, protection of forests and redesign of cities are considered. Finally, reflected on the stabilization of the world population, rules for restructuring the economy and acceleration of the transition process.
\end{abstract}

Keywords: economy; ecology; life preservation. 


\section{Introducción}

La relación existente entre las ciencias económicas y la ecología, debe estrecharse y consolidarse ya que, el crecimiento de la primera y la degradación de la segunda, conspiran contra la estabilidad de una población mundial que de acuerdo a consulta realizada en Pirámide de la Población Mundial (2016) asciende a 7.432.663.00, de los cuales el 50.4\% es masculino $y$ el $49.6 \%$ femenino, estas cifras nos indican, entre otras cosas, que en algunos meses la población mundial estará cercana a los 8 mil millones de habitantes, ocupando espacios en un planeta que no crece, cuyos recursos naturales son escasos, y donde la producción de alimentos no es suficiente para satisfacer los requerimientos mínimos necesarios de cada individuo; como bien nos menciona Fukuyama, (2002) "si se mantienen las pautas de natalidad y mortalidad actuales, en el año 2050 el mundo ofrecerá un aspecto sustancialmente distinto del que presenta hoy". (pág. 104). Y esa distinción se presentará de manera no deseada si no se toman las medidas para garantizar una vida sustentable en el planeta.

Lo anterior nos indica que se deben generar los recursos para que semejante cantidad de personas se sostengan de manera satisfactoria, no es difícil entender que la generación, producción y distribución de recursos requieren de un importante apoyo económico tanto de los gobiernos de las naciones como del sector privado, es aquí donde los ambientalistas creen entonces que la economía es un apéndice o subconjunto del desarrollo, sin embargo los economistas piensan que es precisamente gracias a ellos que se produce el desarrollo, siendo este un subconjunto que se deriva de economías sanas y rentistas.

Para aclarar semejante polémica es preciso decir que la teoría económica y sus leyes no explican el calentamiento global, ni el efecto invernadero tampoco nos dicen las razones del derretimiento de los glaciares y menos explica la desaparición de millones de especies de la faz de la tierra. 
Por lo tanto, pareciese que la economía y los sistemas naturales permanecerán en conflicto, y que el crecimiento de la economía mundial la puede llevar a un declive permanente, ya que el comportamiento de esta no se adapta a las nuevas tendencias del desarrollo.

Para evitar el desastre, ecologistas y economistas deben trabajar unidos en lo que llamaremos la "verderización" de la economía, que no es más que la unión de dos grandes ramas del saber que siempre surcaron caminos distintos y que hora el momento les exige trabajar en conjunto por esa ecoeconomía necesaria sobre todo para garantizar la calidad de vida de los futuros habitantes del planeta. La brecha entre economistas y ecologistas no puede seguir creciendo. Esto no parece tarea fácil debido a que estos profesionales pueden mirar un mismo objeto de estudio de manera muy distinta, por ejemplo, el economista estudia el costo y los beneficios de producir el combustible, el ecologista estudia los daños que esta causa al ambiente. Esta integración, para que sea exitosa, debe tener como premisa la consideración de que, la economía es un subconjunto de la ecología, sin restarle importancia a las ciencias económicas.

\section{Desarrollo}

En este momento es bueno preguntarse ¿Que hacer para relacionar la economía con la ecología como medio para salvar el planeta? Para responder a esto podemos decir que pasamos mucho tiempo preocupándonos por nuestros déficits económicos, pero son los déficits ecológicos los que amenazan nuestra economía en el largo plazo. Los déficits económicos los cubrimos afectando económicamente a nuestros "epocales", pero los ecológicos los trasladamos a las personas del futuro, la transformación de esta economía, ambientalmente destructiva, requiere de un giro Copernicano en nuestra mentalidad económica, y el reconocimiento de que la economía es parte de los ecosistemas y que la tierra solo puede sostener el progreso si la 
economía se reestructura de manera que sea compatible con ella. En este sentido, Naredo (2011) nos dice:

Existen dos posibilidades de hacer que la gestión económica considere esa parte oscura de los procesos. Una, tratando de iluminar los elementos que la componen mediante prácticas de valoración que permitan aplicar sobre ellos el instrumental analítico habitual de los economistas que razona en términos de precios, costes y beneficios monetarios efectivos 0 simulados. Otra, adaptando a las exigencias de la gestión el aparato analítico de disciplinas que, como la ecología o la termodinámica, se preocupaban ya de aclarar lo ocurrido en esa parte oscura del proceso económico. (pág. 237).

Desafortunadamente en la actualidad no se ha desarrollado un marco conceptual que permita este tipo de economía, para ello se hace necesario que se conozcan y sobre todo comprendan algunos términos propios de la ecología tal como, rendimiento sostenible, capacidad de carga, ciclos de nutrientes, ciclo hidrológico y cambio climático, solo por mencionar algunos.

Para darle forma a esta eco-economía se debe establecer una relación entre los científicos ecologistas y los encargados de desarrollar los proyectos económicos de forma tal que los primeros evalúen los impactos ambientales de los grandes proyectos antes de sus ejecución, un economista no entrenado en ecología tiene altas probabilidades de que los proyectos que desarrolle, tengan algún impacto negativo sobre el ambiente; así mismo la economía mundial, en la actualidad, se guía por las tendencias del mercado, no tomando en cuenta los principios de la ecología. Lamentablemente, al no reflejar los costos ecológicos el mercado ofrece información engañosa para los tomadores de decisiones en todos los niveles económicos. Es en base a esto que la economía se mueve según Foladori (2001):

Con ritmos basados exclusivamente en la dinámica de los precios, los cuales se contraponen con los ritmos naturales. Es necesario, por tanto, que la actividad económica contemple los 
diferentes ritmos naturales y, con ello, la distinción entre recursos naturales renovables y no renovables. (pág. 137).

En este sentido el reto seria tomar a la naturaleza como modelo para el diseño de la nueva economía mundial acorde con las demandas del ecosistema más que del mercado.

Hay suficiente evidencia acumulada que muestra que la economía global está siendo lentamente socavada en varias de sus aristas, si queremos continuar con una economía en progreso, no nos queda otro remedio que, de manera sistemática, reestructurar la economía mundial con el fin de que sea ambientalmente sostenible. Esto es una tarea monumental, titánica, majestuosa, es una labor sin precedentes ya que se han realizado muchas transformaciones económicas pero ninguna basada en los principios de la ecología, sobre todo con las proyecciones esperadas del crecimiento económico.

Así para que podamos ver a la eco-economía como un paradigma emergente, se requiere de un cambio sistémico de las señales de mercado acordes con los principios de sostenibilidad ecológica. La reestructuración de la economía, debe pasar por tres aspectos fundamentales, energía, materiales y alimentos; estos aspectos se convierten en las categorías emergentes del nuevo paradigma que llamaremos, "verderización" de la economía; en el caso de la energía pasar de los combustibles fósiles, del carbón y el gas natural, a los aerogeneradores, a las celdas solares y a la geotérmica no es fácil de imaginar. En el caso de los materiales la cosa es distinta ya que, a groso modo, hay que pasar linealmente de la industria de extracción de minerales o de la tala de un bosque, a la industria del reciclaje emulando, como mencionamos anteriormente, a la naturaleza donde nada se desperdicia. En el caso de los alimentos, los grandes cambios no son en su estructura, pero si en el modelo de gestión del sector. 
En el caso de la energía verde, o fuentes alternas de energía, la inversión en investigación y desarrollo para sentar las bases de una energía eficiente, donde los combustibles fósiles deben ser sustituidos por la energía solar, la eólica, hidráulica, geotérmica y/o mareomotriz; los gobiernos del mundo en unión con los centros de investigación públicos y privados, deben apoyar y presentar sus proyectos que permitan la masificación de las energías alternativas para mover al mundo.

En este orden de ideas, la creación de nuevos materiales, y la mejora de los existentes, teniendo como meta el cien por ciento de "reciclabilidad", incentivando fiscalmente a las empresas que reciclen, y aprovechando las bondades de la nanotecnología, debe ser una de las acciones conjuntas de las comunidades y gobiernos del mundo. Se hace énfasis en el reciclaje de materiales en función del impacto positivo que representa una política sobre el tema.

Por otro lado, el derecho a una alimentación adecuada, balanceada y de calidad, debe ser un artículo común en todas las constituciones de las naciones del mundo, en la actualidad según la Organización de las Naciones Unidas para la Agricultura y Alimentación, FAO (2015), más de 1.2 billones de personas en el mundo presentan algún tipo de desnutrición, En su forma primaria, el hambre es un problema netamente de productividad. Por lo general la gente tiene hambre porque no se producen suficientes alimentos, y/o porque no tienen suficientes ingresos para comprarlos.

Las políticas económicas dedicadas a reducir el hambre en el mundo deben incluir necesariamente un control del crecimiento de la población, en ese sentido para Brown (2006), "Demográficamente, la mayoría de los pobres del mundo viven en países con poblaciones en rápido crecimiento, donde la pobreza y el crecimiento demográfico se refuerzan entre sí" (pág. 147). Esto es un indicativo de que, para garantizar la alimentación de la población 
mundial, esta debe controlar su crecimiento, con estrategias para controlar la fertilidad, campañas educativas, y de divulgación.

Adicionalmente, se debe considerar de manera muy seria las consecuencias que el cambio climático trae sobre la productividad global de alimentos, para Cabrera (2014) "el aumento de la temperatura promedio del planeta para el año 2100 estará entre $1,4^{\circ}$ y $4,8^{\circ}$ C", (pág. 85), tal incremento tendrá incidencia directa sobre la producción, disponibilidad, estabilidad del mercado de alimentos, acceso a alimentos y utilización de los mismos. Esto debe considerarse no como un riesgo futuro ya que el cambio climático está en pleno desarrollo, paradójicamente los países que menos contribuyen con los efectos negativos del calentamiento global y el cambio climático son los más afectados por sus consecuencias, en ese sentido en el informe sobre La Cumbre Mundial de Seguridad Alimentaria, realizada en Roma en 2009 se concluye entre otras cosas que "es probable que tal efecto sea inmediato y grave sobre la seguridad alimentaria en el hemisferio Sur, donde la oferta alimentaria podría ser notablemente inferior a la actual, ya de por sí insuficiente" (pág. 16).

\section{Reflexiones Finales}

Estamos convencidos de que se deben hacer cambios importantes en la economía mundial y muchos de estos cambios deben comenzar a realizarse ahora mismo, aunque a mayoría de las escuelas de pensamiento económico reconocen, que en la actualidad existe una crisis ambiental, aunque discrepen en el grado de profundidad y en las medidas correctivas que se deben tomar, la construcción de una eco-economía afectará a todas las facetas de nuestras vidas. Estos cambios alterarán la forma en que encendemos nuestros hogares, lo que comemos, como nos trasladamos, cómo usamos nuestro tiempo de ocio, y el número de hijos que tenemos. Eso nos dará un mundo donde somos parte de la naturaleza, en lugar de sentirnos distanciados de ella. 
Una economía que no está en sintonía con el ecosistema del planeta se presenta antagónica y profundamente contaminante, perjudicial, y en última instancia autodestructiva, la base actual, es decir los combustibles fósiles, y la economía de usar y botar, debe cambiar para preservar la vida en el planeta. En el mundo occidental se ha elevado el nivel de vida de una quinta parte de la humanidad a unos niveles que nuestros antepasados jamás podían imaginar, proporcionando una dieta muy variada, con nuevos materiales y una movilidad física que ni el mismo $\mathrm{Da}$ Vinci pudo imaginar. Pero desgraciadamente, en un futuro no muy lejano, esto no funcionará para esa mencionada quinta parte de la población mucho menos para el mundo entero.

Tres grandes categorías deben emerger con miras a salvaguardar la vida en el planeta, la primera se refiere a un cambio radical en la forma de transformar la energía llevando a niveles mínimos el uso de los combustibles fósiles permitiendo de esta manera frenar los efectos negativos del cambio climático; la otra se refiere a una política de reciclaje que busque materiales que permitan su rehúso de manera indefinida, dando descanso a la explotación natural y la contaminación natural, la última y más importante, se refiere a la garantía de una alimentación con la calidad y cantidad adecuada para la totalidad de la población mundial, aumentando la productividad de los distintos rubros alimenticios, acompañados con políticas proteccionistas del ambiente; este paradigma emergente debe estar acompañado, para ser exitoso, de un control de la fertilidad, que permita mantener niveles de población mundial acorde con los niveles productivos, Según cifras de la organización de las Naciones Unidas para la Alimentación y Agricultura (FAO), para el año 2050 habrán en el mundo 9.100.000.000 millones de habitantes que deben ser alimentados de manera adecuada, lo cual si no se toman las medidas adecuadas se convertirá en una tarea muy difícil de cumplir. 


\section{Referencias}

Brown, L. (2006). Plan B Rescatando un Planeta estresado y una Civilización en Problemas. NY: WW, Norton \& Co.

Cabrera, O. (2014). Fuentes Alternas de Energía. UBV. Caracas.

FAO (2009). Cumbre Mundial Sobre la seguridad Alimentaria 2009. [Documento en línea]. Recuperado de: http://www.fao.org/wsfs/cumbre$\underline{\text { mundial/es }}$

Foladori, G. (2001). Controversias Sobre Sustentabilidad, la coevolución sociedad- naturaleza. Universidad Autónoma de Zacatecas.

Fukuyama, F. (2002). El fin del hombre: Consecuencias de la Revolución Biotecnológica. Barcelona: Ediciones B.

Naredo, J. (2011). Fundamentos de Economía Ecológica. Edición electrónica revisada, CIP Ecosocial.

Pirámide de la Población Mundial (2016). Población: Mundo 2016. PopulationPyramid.net [Documento en línea]. Recuperado de: https://populationpyramid.net/es/mundo/2016/ 


\section{Oscar Eduardo Cabrera Venot \\ e-mail: oscarcvenot@gmail.com}

Nacido en Venezuela. Ingeniero Mecánico (1983)

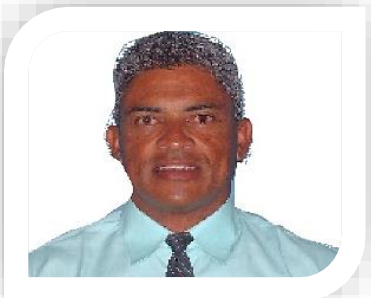

Magister en Economía de Empresas (1990),

Magister en Gerencia de Mantenimiento (2007),

Doctor en Ciencias de la educación 2014,

Posdoctorado en estudios libres 2016.

Gerente de ventas en Honda de Venezuela, Gerente de Compras en grupo Mavesa, vopack, Docente universitario, Actualmente Jefe de investigación, extensión y postgrado IUTPC.

El contenido de este manuscrito se difunde bajo una Licencia de Creative Commons ReconocimientoNoComercial-Compartirlgual 4.0 Internacional 